\title{
Response to immunomodulatory drug-based regimens in heavily pretreated patients with recurrent/refractory adult Langerhans cell histiocytosis
}

\section{Xin-xin Cao}

Peking Union Medical College Hospital https://orcid.org/0000-0001-7884-3073

Ai-lin Zhao

Peking Union Medical College Hospital

Xue-min Gao

Peking Union Medical College Hospital

Hui-lei Miao

Peking Union Medical College Hospital

Hong-xiao Han

Peking Union Medical College Hospital

$\mathrm{Na}$ Niu

Peking Union Medical College Hospital

Jian Li

Peking Union Medical College Hospital

Dao-bin Zhou

Peking Union Medical College Hospital

Minghui Duan ( $\nabla$ mhduan@sina.com )

https://orcid.org/0000-0003-2130-6300

\section{Research article}

Keywords: Langerhans cell histiocytosis, adult, recurrent/refractory, immunomodulatory drug-based regimen

Posted Date: December 6th, 2019

DOl: https://doi.org/10.21203/rs.2.18343/v1

License: (a) (1) This work is licensed under a Creative Commons Attribution 4.0 International License. Read Full License 


\section{Abstract}

Purpose: Langerhans cell histiocytosis (LCH) is a clonal histiocytic neoplasm and, because of its rarity in adults, there is no standard treatment for adult LCH. Immunomodulatory drugs (IMiDs) have been used to treat patients with low risk recurrent/refractory LCH but their effectiveness in adult LCH patients is unclear.

Methods: We retrospectively evaluated the response rate to IMiDs-based regimens in ten heavily pretreated recurrent/refractory adult LCH patients at Peking Union Medical College Hospital.

Results: A total of 10 patients (four males and six females) were included in this study. Median age at diagnosis was 33 years (range, 28-54 years). All patients had multisystem involvement and the median number of organs involved was 5 (range, 5-7). Seven patients had high risk organs involved, including seven patients with liver involvement and one with spleen involvement. All 10 patients had received at least one chemotherapy before the IMiDs-based regimen. The median number of previous lines of chemotherapy was 2 (range, 1-4). Eight patients received thalidomide, dexamethasone and cyclophosphamide, and two patients received lenalidomide and dexamethasone. The median time that patients received thalidomide treatment was 15 months and the duration of the two patients on lenalidomide regimen was 3 months and 12 months separately. Treatment responses in eight recurrent $\mathrm{LCH}$ patients included non-active disease in one patient and regressive disease in seven patients. The two refractory patients who had progression during the last treatment had stable disease after IMiDs therapy. During a median 15-months follow-up period, no disease reactivation or death was observed.

Conclusions: IMiDs combined with dexamethasone and cyclophosphamide, may be a salvage therapy for recurrent/refractory adult LCH patients.

\section{Introduction}

Langerhans cell histiocytosis $(\mathrm{LCH})$ is a rare, heterogeneous histiocytic disorder occurring most commonly in children.[1] The clinical presentation and outcome of $\mathrm{LCH}$ are extremely variable, ranging from isolated, spontaneously remitting pulmonary LCH to multisystem disease, with life-threatening organ dysfunction. LCH lesions are defined histologically by langerin-positive (CD207 $)$ histiocytes on an inflammatory background that includes lymphocytes, eosinophils and macrophages.[2] There is substantial evidence, including the discovery of the BRAF ${ }^{\mathrm{V} 600 \mathrm{E}}$ mutation in $\mathrm{LCH}$ in 2010 , that $\mathrm{LCH}$ is driven by pathological MAPK activation in myeloid precursors.[3, 4] LCH is now believed to have both inflammatory and neoplastic characteristics and is designated an "inflammatory myeloid neoplasm". [5]

The current standard treatment protocol for children with de novo multisystem LCH is vinblastine plus prednisone. [6] Because of the rarity of LCH in adults and a lack of prospective randomized trials, the treatment strategy for adults is mostly based on pediatric protocols. The overall response rate of therapy based on vinblastine plus prednisone in adults is lower than in children and the treatment tends to show higher toxicity. [7] A retrospective study showed an advantage for cytarabine monotherapy compared with 
vinblastine/prednisone in bone LCH patients. [8] Although the overall survival in adult LCH was over 2 decades, disease recurred at least once in $40 \%$ of patients. $[9,10]$ There is little data to guide therapy after frontline treatment has failed. Nucleoside analogues may be a reasonable class of drugs to treat $\mathrm{LCH}$, but are associated with prolonged hospitalization and high rates of treatment-related death. [11, 12] A retrospective study from Mayo showed cladribine and vemurafenib, a BRAF inhibitor, had an overall response rate of 50-69\%. [13] Immunomodulatory drugs (IMiDs), such as thalidomide and lenalidomide, strongly boost the immune response and are widely used to treat plasma cell diseases. In a phase 2 trial, thalidomide as monotherapy gave a $70 \%$ response rate in recurrent/refractory low risk LCH but there were no responses in six high risk children. [14] IMiDs combined with dexamethasone have been successfully used to treat high risk children as well as adult LCH patients. $[15,16]$ Cyclophosphamide has been used as part of combination chemotherapy regimens to treat adult LCH, such as such as MACOP-B (methotrexate, doxorubicin, cyclophosphamide, vincristine, bleomycin and prednisone). [17]

We have, therefore, retrospectively analyzed the efficacy and toxicity of IMiDs combined with dexamethasone and cyclophosphamide regimens in the treatment of recurrent/refractory LCH among adult patients at our hospital.

\section{Methods}

\section{Patients}

A retrospective review was conducted among patients who were diagnosed with $\mathrm{LCH}$ and had received thalidomide- or lenalidomide-based therapy for at least 3 months at Peking Union Medical College Hospital between January 2016 and March 2019. Patients who were younger than 18-years-old were excluded from the study. Diagnosis of $\mathrm{LCH}$ was based on histological findings that were reviewed independently by two pathologists. Informed consent was obtained from all patients and the protocol was approved by the Peking Union Medical College Hospital Ethics Committee. The study was performed in accordance with the ethical standards of the 1964 Declaration of Helsinki and its later amendments. Age, sex, lesion location, physical examination results, routine biologic analyses, treatment and survival were recorded for all study participants. Risk organs and organ involvement were recorded as defined by Histiocyte Society criteria. [6]

\section{Treatment}

All patients received an IMiDs-based regimen as follows. Thalidomide $(100 \mathrm{mg})$ was administered every night and dexamethasone (20 or $40 \mathrm{mg}$ daily) was administered on days 1, 8, 15 and 22, with

cyclophosphamide $\left(300 \mathrm{mg} / \mathrm{m}^{2}\right)$ on days 1,8 and 15 of a 28-day cycle (TCD). Lenalidomide $(25 \mathrm{mg})$ was administered on days 1 to 21 and dexamethasone (20 or $40 \mathrm{mg}$ daily) was administered on days 1, 8, 15 and 22 of a 28-day cycle (RD). Treatment continued until disease progression or unacceptable side effect. All patients received aspirin thromboprophylaxis during IMiDs treatment.

\section{Response criteria}


All patients were followed up every 3 months. The assessment was performed by laboratory tests, clinical assessment, physical examination and imaging as appropriated for the LCH location. Imaging data were collected by 18F-fluorodeoxyglucose positron emission tomography (FDG-PET) from 2 patients, thoracic high resolution computed tomography (HRCT) and cerebral magnetic resonance imaging (MRI). Disease was assessed using standard evaluations, as defined by Histiocyte Society criteria. [6] If all signs and symptoms were resolved, the patients were classified as having non-active disease (NAD). Otherwise, they were classified as having active disease (AD). AD was further subdivided into regressive disease (signs and symptoms were improved with no new lesions), stable disease (persistence of signs and symptoms) or progressive disease (progression and/or appearance of new lesions). The response was assessed by review of records.

\section{Data analysis}

Overall survival (OS) was defined as the duration from initiation of treatment with thalidomide- or lenalidomide-based therapy to the date of death or last follow-up. Progression-free survival (PFS) was calculated from the date of initiation of thalidomide- or lenalidomide-based therapy until the date of disease progression, relapse, death from any cause or last follow-up.

\section{Data sharing statement}

Individual participant data will not be available

\section{Results}

\section{Patient Characteristics}

A total of 10 patients (four males and six females) were included in this retrospective study. Median age at diagnosis was 33 years (range, 28-54 years). All patients had multisystem involvement and the median number of organs involved was 5 (range, 5-7). Detailed information for the patients included in this study is shown in Table 1. Seven patients had a high risk organ involved, including seven with liver involvement and one with spleen involvement (Patient 5 had both liver and spleen involved). The most commonly affected organs were lung (9/10), pituitary $(9 / 10)$, bone $(8 / 10)$, liver $(7 / 10)$ and lymph node (7/10). All 10 patients had received at least one prior of chemotherapy before the IMiDs-based regimen. The median number of previous lines of chemotherapy was 2 (range, 1-4). All the patients had relapsed or refractory to the initial therapies, which included cytarabine monotherapy $(2 / 10)$, cytarabine combined with methotrexate (8/10), vindesine and prednisone-based regimen (4/10), cladribine monotherapy $(2 / 10)$ or cladribine combined with cytarabine (2/10). One patient had a liver transplantation because of severe involvement of the liver. The median time from diagnosis to the initiation of IMiDs-based treatment was 29.5 months (range, 4-96 months). Before the IMiDs-based regimens, eight patients had recurrent LCH and two patients had progression during the last treatment (Patients 9 and 10). 
Eight patients with recurrent/refractory LCH received TCD. The median time that patients received thalidomide treatment was 15 months (range, 5-24 months). Two patients received RD, one for 3 months and one for 12 months. In the eight recurrent LCH cases, treatment led to NAD in one patient and regressive disease in seven patients. The two patients who had progression during the last treatment had stable disease after receiving IMiDs-based treatment for 3 and 14 months.

Of the seven patients who had liver involvement, $5 / 6$ (83.3\%) had elevated levels of alkaline phosphatase (ALP), 6/6 had elevated levels of $y$-glutamyl transpeptidase (GGT) and 5/7 (71.4\%) had elevated levels of total bilirubin (Tbil) before initiation of the IMiDs-based regimen. After treatment, the median level of ALP dropped from $284 \mathrm{U} / \mathrm{L}$ (range, 104-627 U/L) to $134 \mathrm{U} / \mathrm{L}$ (range, 92-325 U/L) (Figure 1). The level of serum GGT dropped from 534.5 U/L (range, 100-1003 U/L) to $107 \mathrm{U} / \mathrm{L}$ (range, 21-270 U/L) and the level of Tbil decreased from $29.1 \mathrm{umol} / \mathrm{L}$ (range, 6.6-145.6 umol/L) to $16.9 \mathrm{umol} / \mathrm{L}$ (range, $7.8-32.2 \mathrm{umol} / \mathrm{L}$ ).

In term of adverse events, one patient developed septic shock caused by infectious diarrhea during the treatment (Patient 2). After the infection was controlled, the patient continued TCD treatment without further infections. Two patients had grade 1 peripheral neuropathy (Patients 1 and 6) and two patients had grade 2 hyperglycemia (Patients 2 and 3). Patients 2 and 3 discontinued dexamethasone after 11 and 18 months of TCD treatment, respectively, because of hyperglycemia but continued with thalidomide and cyclophosphamide therapy.

\section{Follow-up}

The median follow-up time for this cohort was 15 months (range, 3-25 months). No disease reactivation or death was observed. The median PFS and OS were not reached yet. One patient had oral squamous cell carcinoma (Patient 3).

\section{Discussion}

$\mathrm{LCH}$ is a heterogeneous disease, the clinical manifestations of which are related to the pattern of organ involvement. Patients with localized disease in "low risk" organs typically have a good prognosis whereas multisystem disease, especially involving "high risk" organs (liver, spleen and bone marrow) increases the likelihood of poor outcomes and disease reactivation following frontline therapy. $[1,6]$ There are no universally accepted standard guidelines for the treatment of adult LCH patients. The majority of treatment reports are retrospective case studies based on small cohorts. There are even fewer reports describing treatment of recurrent/refractory disease. In this retrospective study, we have demonstrated the efficacy of IMiDs combined with dexamethasone and cyclophosphamide, in recurrent/refractory LCH patients.

Thalidomide monotherapy has been used to treat LCH in low risk patients but failed in children with high risk LCH. [14] In our cohort, seven patients had liver involvement and one patient also had spleen involvement. All of the patients had relapsed or refractory to initial therapies, including cytarabine. Four patients had failed on a vindesine and prednisone-based regimen and three on a cladribine-based 
regimen. Our patients thus had severe $\mathrm{LCH}$ and had been heavily pretreated before the IMiDs-based treatments. After received TCD or RD treatment, $10 \%$ of patients had NAD, $70 \%$ of patients had regressive disease and $20 \%$ of patients had stable disease. During a median of 15 -months follow-up, no disease reactivation or death was observed. Our use of doublet or triplet regimens instead of monotherapy may explain why our results differ from the earlier reports. [14] Another reason may be that the patients involved in our cohort were all adult patients instead of children.

The pathogenesis of $\mathrm{LCH}$ remains largely obscure. The current understanding is that $\mathrm{LCH}$ is an inflammatory myeloid neoplasm, driven by pathological MAPK pathway activity. The hypothesis is that langerin-positive histiocytes, harboring an oncogenic mutation, migrate to the site of lesions, where they recruit and activate various inflammatory cells ${ }^{5}$. In LCH lesions, the pathological histiocytes constitute approximately $8 \%$ of the granulomatous lesion. [18] The remainder of the lesion is composed of inflammatory infiltrate, including activated T cells on the background of a cytokine storm. [19] Studies of other conditions have demonstrated that the MAPK pathway does influence tumor necrosis factor (TNF)a function. [20,21] IMiDs inhibit the production of TNF-a,[22] inhibit the activity of NF-KB[23] and have anti-inflammatory effects. [24] IMiDs have also been shown to act synergistically with dexamethasone, both in vitro and in vivo [25] in mantle cell lymphoma. These data led to the hypothesis that IMiDs combined with dexamethasone and cyclophosphamide, might be a promising treatment for recurrent/refractory adult LCH patients, as shown in our cohort.

Since recurrent somatic activating mutations of $\mathrm{BRAF}^{\mathrm{V} 600 \mathrm{E}}$ and additional genetic drivers of ERK pathway activation have been identified. [3, 4] There were small studies demonstrated the efficacy of targeted therapies, BRAF or MEK inhibitors in LCH. $[12,26]$ Monotherapy might be better tolerated than triplet regimens, but prospective studies should be designed to test the efficacy and toxicity of targeted therapies in adult $\mathrm{LCH}$.

This study has several limitations. First, it is a single-institution retrospective study and the number of subjects enrolled in the study was relatively small, which might limit the generalizability of our results. However, all the records were reviewed independently by two hematologists to minimize bias or errors in data collection. Second, BRAF mutation status were not available in this cohort. Most of patients did not have an adequate amount of tissue available for genomic testing. Lastly, since we used doublet or triplet regimens in this study, it is difficult to conclude the treatment result were from IMiDs. An ongoing clinical trial at Dana Farber for lenalidomide monotherapy in adult LCH patients (NCT02523040) may provide more data at this point. And further prospective study may be needed to confirm the advantage of IMiDsbased therapy for the treatment of adult LCH patients.

\section{Conclusion}

In conclusion, IMiDs combined with dexamethasone and cyclophosphamide, could provide a useful salvage therapy, with a favorable safety profile, for recurrent/refractory adult LCH patients. 


\section{Declarations}

\section{Compliance with ethical standards}

\section{Conflict of interest}

The authors declare that they have no competing interests.

\section{Ehtics approval and consent to participate}

This study was approved by Peking Union Medical College Hospital Ethics Committee.

\section{Informed consent}

Informed consent was obtained from all patients

\section{Funding}

Institutional research funding provided by the Non-profit Central Research Institute Fund of the Chinese Academy of Medical Sciences (2019-RC-HL-001, for CXX), Institutional research funding was provided by the CAMS Innovation Fund for Medical Sciences (Grant No. 2016-12M-1-002, for LJ), and The National Key Research and Development Program of China (Grant No. 2016YFC0901503, for LJ).

\section{References}

1. Stockschlaeder M, Sucker C (2006) Adult Langerhans cell histiocytosis. Eur J Hematol 76:363-368.

2. Chikwava K, Jaffe R (2004) Langerin (CD207) staining in normal pediatric tissues, reactive lymph nodes, and childhood histiocytic disorders. Pediatr Dev Pathol 7: 607-614.

3. Badalian-Very G, Vergilio JA, Degar BA, et al (2010) Recurrent BRAF mutations in Langerhans cell histiocytosis. Blood 116: 1919-1923.

4. Chakraborty R, Hampton OA, Shen X, et al (2014) Mutually exclusive recurrent somatic mutations in MAP2K1 and BRAF support a central role for ERK activation in LCH pathogenesis. Blood 124: 30073015.

5. Berres ML, Allen CE, Merad M.Adv (2013) Pathological consequence of misguided dendritic cell differentiation in histiocytic diseases. Adv. Immunol 120: 127-161.

6. Gadner H, Minkov M, Grois N, et al (2013) Therapy prolongation improves outcome in multisystem Langerhans cell histiocytosis. Blood 121: 5006-5014.

7. Kobayashi M, Tojo A. Langerhans cell histiocytosis in adults: Advances in pathophysiology and treatment. Cancer Sci. 2018; 109: 3707-3713.

8. Cantu MA, Lupo PJ, Bilgi M, et al (2012) Optimal therapy for adults with Langerhans cell histiocytosis bone lesions. PLoS One 7: e43257. 
9. Goyal G, Shah MV, Hook CC, et al (2018) Adult disseminated Langerhans cell histiocytosis: incidence, racial disparities and long-term outcomes. $\mathrm{Br} \mathrm{J}$ Haematol 182: 579-581.

10. Minkov M, Steiner M, Po tschger U, et al (2008) International LCH Study Group. Reactivations in multisystem Langerhans cell histiocytosis: data of the international LCH registry. J Pediatr 153: 700705.

11. Donadieu J, Bernard F, van Noesel M, et al (2015) Cladribine and cytarabine in refractory multisystem Langerhans cell histiocytosis: results of an international phase 2 study. Blood 126: 1415-1423.

12. F, Thomas C, Bertrand $Y$, et al (2005) Multicentre pilot study of 2-chlorodeoxyadenosine and cytosine arabinoside combined chemotherapy in refractory Langerhans cell histiocytosis with haematological dysfunction. Eur J Cancer 41: 2682-2689.

13. Goyal G, Hu M, Young JR et al (2019) Adult Langerhans cell histiocytosis: A contemporary singleinstitution series of 186 patients. J Clin Oncol 37 (suppl; abstr 7018).

14. McClain KL, Kozinetz CA (2007) A phase II trial using thalidomide for Langerhans cell histiocytosis. Pediatr Blood Cancer 48: 44-49.

15. Uppuluri R, Ramachandrakurup S, Balaji R, et al (2017) Successful treatment of refractory Langerhans cell histiocytosis of the choroid plexus in a

16. child with pulse dexamethasone and lenalidomide. J Pediatr Hematol Oncol 39: e74-e78.

17. Adam Z, Rehák Z, Koukalová R, et al (2012) Lenalidomide induced therapeutic response in a patient with aggressive multi-system Langerhans cell histiocytosis resistant to 2-chloro-deoxyadenosine and early relapsing after high-dose BEAM chemotherapy with autologous peripheral blood stem cell transplantation. Vnitr Lek 58: 62-71.

18. Derenzini E, Stefoni V, Pellegrini C, et al (2015) High efficacy of the MACOP-B regimen in the treatment of adult Langerhans cell histiocytosis, a 20 year experience. BMC Cancer 15: 879.

19. Berres ML, Lim KP, Peters T, et al (2014) BRAF-V600E expression in precursor ver- sus differentiated dendritic cells defines clinically distinct LCH risk groups. J Exp Med 211: 669-683.

20. Morimoto A, Oh Y, Nakamura S, et al (2017) Inflammatory serum cytokines and chemokines increase associated with the disease extent in pediatric Langerhans cell histiocytosis. Cytokine 97: 73-79.

21. Menon MB, Gaestel M (2018) MK2-TNF-signaling comes full circle. Trends Biochem Sci 43: 170-179.

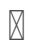

22. Ray AL, Berggren KL, Restrepo CS, et al (2018) Inhibition of MK2 suppresses IL-1b, IL-6, and TNF-adependent colorectal cancer growth. Int J Cancer 142: 1702-1711.

23. Sampaio EP, Sarno EN, Galilly R, Cohn ZA and Kaplan G (1991) Thalidomide selectively inhibits tumor necrosis factor alpha production by stimulated human monocytes. J Exp Med 173: 699-703.

24. Yagyu T, Kobayashi H, Matsuzaki H, Wakahara K, Kondo T, Kurita N and Sekino H, et al (2005) Thalidomide inhibits tumor necrosis factor- alpha-induced interleukin-8 expression in endometriotic stromal cells, possibly through suppression of nuclear factor-kappaB activation. J Clin Endocrinol Metab 90: 3017-3021. 
25. Fujimoto H, Noguchi T, Kobayashi H, Miyachi H and Hashimoto Y (2006) Effects of immunomodulatory derivatives of thalidomide (IMiDs) and their analogs on cell-differentiation, cyclooxygenase activity and angiogenesis. Chem Pharm Bull (Tokyo) 54: 855-860.

26. Qian Z, Zhang L, Cai Z, Sun L et al (2011) Lenalidomide synergizes with dexamethasone to induce growth arrest and apoptosis of mantle cell lymphoma cells in vitro and in vivo. Leuk Res 35: 380-386.

27. Diamond EL, Durham BH, Ulaner GA, et al (2019) Efficacy of MEK inhibition in patients with histiocytic neoplasms. Nature 567: 521-524.

\section{Table}

Table 1. Patients' characteristics and treatment responses

\begin{tabular}{|c|c|c|c|c|c|c|c|c|c|c|}
\hline No & $\begin{array}{l}\text { Sex/ } \\
\text { age }\end{array}$ & $\begin{array}{l}\text { Involved } \\
\text { organs }\end{array}$ & $\begin{array}{l}\text { Prior } \\
\text { treatement }\end{array}$ & $\begin{array}{l}\text { Prior } \\
\text { lines of } \\
\text { therapy }\end{array}$ & $\begin{array}{l}\text { TDTI } \\
\text { (m) }\end{array}$ & $\begin{array}{l}\text { IMiDs } \\
\text { regimen }\end{array}$ & $\begin{array}{l}\text { Time } \\
\text { on } \\
\text { IMiDs } \\
\text { (m) }\end{array}$ & Response & $\begin{array}{l}\text { PFS } \\
(\mathrm{m})\end{array}$ & $\begin{array}{l}\text { OS } \\
(\mathrm{m})\end{array}$ \\
\hline 1 & $\mathrm{~F} / 51$ & $\begin{array}{l}\text { B, LN, } \\
\text { S, M, P }\end{array}$ & $\begin{array}{l}\text { CEOP, } \\
\text { MA, AP, } \\
\text { AraC }\end{array}$ & 4 & 54 & TCD & 15 & ReD & 15 & 15 \\
\hline 2 & $\mathrm{~F} / 28$ & $\begin{array}{l}\text { P, S, } \\
\text { LN, T, L }\end{array}$ & $\begin{array}{l}\text { CEOP, } \\
\text { GDPM, } \\
\text { AraC }\end{array}$ & 3 & 60 & TCD/TC & $11 / 12$ & NAD & 24 & 24 \\
\hline 3 & M/31 & $\begin{array}{l}\text { S, P, L, } \\
\text { B, LN, } \\
\text { M }\end{array}$ & $\mathrm{MA}$ & 1 & 30 & $\mathrm{TCD} / \mathrm{TC}$ & $18 / 6$ & ReD & 25 & 25 \\
\hline 4 & $\mathrm{~F} / 46$ & $\begin{array}{l}\text { P, L, B, } \\
\text { Li, LN }\end{array}$ & MA & 1 & 24 & $\mathrm{RD}$ & 12 & ReD & 12 & 12 \\
\hline 5 & M/33 & $\begin{array}{l}\text { P, L, B, } \\
\text { LN, S, } \\
\text { Li, Sp }\end{array}$ & MA, CEOP & 2 & 29 & TCD & 15 & ReD & 15 & 15 \\
\hline 6 & $\mathrm{~F} / 54$ & S, P, L, & MA & 1 & 9 & TCD & 21 & $\operatorname{ReD}$ & 21 & 21 \\
\hline 7 & $\mathrm{M} / 31$ & P, L, Li, & MA & 1 & 4 & TCD & 12 & $\operatorname{ReD}$ & 12 & 12 \\
\hline 8 & $\mathrm{~F} / 49$ & $\mathrm{P}, \mathrm{L}, \mathrm{B}$ & $\mathrm{MA}, \mathrm{CA}, \mathrm{C}$ & 2 & 12 & TCD & 5 & ReD & 5 & 5 \\
\hline 9 & $\mathrm{~F} / 33$ & $\begin{array}{l}\text { L, B, T, } \\
\text { LN, Li }\end{array}$ & $\begin{array}{l}\text { CVD, } \\
\text { CHOPE, } \\
\text { CA, C }\end{array}$ & 3 & 55 & TCD & 14 & SD & 14 & 14 \\
\hline 10 & $\mathrm{M} / 34$ & $\begin{array}{l}\text { P, L, Li, } \\
\text { B }\end{array}$ & MA, C, LiT & 2 & 96 & $\mathrm{RD}$ & 3 & SD & 3 & 3 \\
\hline
\end{tabular}

TDTI, time from diagnosis to IMiDs; IMiDs, immunomodulatory drugs; $B$, bone; LN, lymph nodes; $S$, skin; M, mastoid; P, pituitary; L, lung; T, thyroid; Li, liver; Sp, spleen; CEOP, cyclophosphamide, etoposide, vindesine and prednisone; MA, methotrexate and cytarabine; AP, cytarabine and etoposide; AraC, cytarabine; TCD, thalidomide, cyclophosphamide and dexamethasone; GDPM, gemcitabine, dexamethasone, cisplatin and methotrexate; TC, thalidomide and cyclophosphamide; $\mathrm{CA}$, cladribine and cytarabine; C, cladribine; EOD, etoposide, vindesine and dexamethasone; CHOPE, cyclophosphamide, doxorubicin, vindesine, prednisone and etoposide; LiT, liver transplantation; ReD, regressive disease; SD, stable disease. NA, not available 